\title{
Effets atomiques sur les émissions bêta à basse énergie : calcul et mesure
}

\section{Atomic effects on beta emissions at low energy : calculation and measurement}

\author{
Martin LOIDL, Xavier MOUGEOT, Marie-Martine BÉ, Charlène BISCH, Cindy LE-BRET \\ et Matias RODRIGUES
}

CEA, LIST, Laboratoire national Henri Becquerel (LNE-LNHB), 91191 Gif-sur-Yvette Cedex, France, martin.loidl@cea.fr

\section{Résumé}

Dans cet article nous présentons les travaux effectués au LNELNHB visant à une meilleure connaissance de la forme des spectres en énergie d'émission bêta : un programme de calcul théorique des spectres a été développé et des spectres ont été mesurés à l'aide de détecteurs cryogéniques. Le calcul théorique inclut une correction de l'effet d'écrantage qui évite la discontinuité non physique inhérente à la méthode de correction habituelle, ainsi qu'une correction de l'effet d'échange, non prise en compte dans la plupart des calculs et importante dans les cas du ${ }^{63} \mathrm{Ni}$ et du ${ }^{241} \mathrm{Pu}$. Ces calculs sont comparés aux spectres expérimentaux obtenus avec des calorimètres métalliques magnétiques, des détecteurs cryogéniques dont l'absorbeur enferme l'émetteur bêta. Ces détecteurs sont caractérisés par un rendement de détection très élevé, une excellente résolution en énergie et un seuil en énergie très bas. Les spectres mesurés du ${ }^{63} \mathrm{Ni}$ et du ${ }^{241} \mathrm{Pu}$ confirment la justesse des spectres théoriques et notamment du calcul de l'effet d'échange jusqu'aux plus basses énergies, explorées pour la première fois.

MOTS CLÉS : SPECTROMÉTRIE BÊTA, CALCUL DE SPECTRES BÊTA, ÉCRANTAGE, EFFET D'ÉCHANGE, DÉTECTEUR CRYOGÉNIQUE, CALORIMÈTRE MAGNÉTIQUE.

\footnotetext{
Abstract

In this paper we present the developments conducted at LNE$L N H B$ with the aim of improving the knowledge of the shapes of beta spectra, concerning the theoretical calculation of the spectra as well as their experimental determination by means of cryogenic detectors. The theoretical calculation comprises a screening correction that avoids the non-physical discontinuity present in the standard correction, as well as a correction of the exchange effect that is usually not taken into account and has a strong impact on the spectra of ${ }^{63} \mathrm{Ni}$ and ${ }^{241} \mathrm{Pu}$. These calculations are compared with experimental spectra obtained with metallic magnetic calorimeters, a class of cryogenic detectors. The beta emitter is enclosed in the absorber of these detectors which offer high detection efficiency, high energy resolution and a very low energy detection threshold. The measured spectra of ${ }^{63} \mathrm{Ni}$ and ${ }^{241} \mathrm{Pu}$ confirm the
}

correctness of the theoretical spectra, in particular the calculation of the exchange effect down to very low energies that have been explored for the first time.

KEYWORDS: BETA SPECTROMETRY, BETA SPECTRA CALCULATION, SCREENING, EXCHANGE EFFECT, CRYOGENIC DETECTOR, METALLIC MAGNETIC CALORIMETER.

\section{Introduction}

Depuis de nombreuses années, les utilisateurs formulent une demande pour une connaissance précise de la forme des spectres en énergie des émissions bêta des radionucléides obéissant à ce mode de désintégration, assortie d'incertitudes bien établies. Cette répartition de la probabilité d'émission des électrons bêta en fonction de l'énergie sera dénommé spectre bêta - ou spectre - dans l'ensemble de cet article. Ces spectres sont nécessaires en métrologie des rayonnements ionisants, dans le milieu médical pour la radiothérapie et la dosimétrie, ainsi que pour les industriels du nucléaire pour les calculs de puissance résiduelle ou la gestion du combustible après irradiation. Ils sont aussi utiles aux institutionnels désireux d'établir un protocole de surveillance des installations nucléaires mondiales grâce à la mesure des neutrinos produits au cœur des réacteurs, tout en permettant d'estimer la quantité produite de matériaux fissiles à usages militaires [1].

Pour les transitions bêta de faible énergie maximale, la connaissance précise de la forme des spectres bêta est essentielle aux mesures d'activité par scintillation 
liquide ou aux calculs de doses déposées. Dans ces deux exemples, un électron bêta de $2 \mathrm{keV}$ aura une influence plus faible que deux électrons de $1 \mathrm{keV}$ chacun. Cela se comprend bien avec le concept de transfert d'énergie linéaire [2]. Dans ce contexte, il est impératif de tenir compte des effets atomiques car ces derniers ont une influence majeure sur la forme du spectre à basse énergie.

Les calorimètres métalliques magnétiques constituent une technique expérimentale puissante pour étudier les spectres en énergie des particules bêta ayant une énergie maximale inférieure à $1 \mathrm{MeV}$. Les spectres bêta du ${ }^{63} \mathrm{Ni}$ [3] et du ${ }^{241} \mathrm{Pu}$ [4] ont été récemment mesurés à l'aide de tels détecteurs. La faible énergie maximale dans chacune de ces deux transitions, couplée à la résolution et au seuil en énergie exceptionnels de ces mesures, permet de tester les prédictions théoriques avec une précision jamais atteinte à ce jour. Le présent article se propose de détailler à la fois les mesures et les calculs théoriques qui ont été réalisés au LNE-LNHB pour ces deux transitions.

\section{Calculs des spectres bêta}

Lors de la désintégration bêta d'un radionucléide X, l'énergie disponible se répartit entre l'électron $\left(\mathrm{e}^{-}\right)$, l'antineutrino $\left(\bar{v}_{\mathrm{e}}\right)$ et le noyau fils $(\mathrm{Y})$ :

$$
{ }_{\mathrm{Z}}^{\mathrm{A}} \mathrm{X} \rightarrow{ }_{\mathrm{Z}+1}^{\mathrm{A}} \mathrm{Y}+\mathrm{e}^{-}+\bar{v}_{\mathrm{e}}
$$

Cette désintégration à trois corps conduit à un spectre en énergie continu pour les électrons. La forme de ce spectre est notamment influencée par la nature de la transition, qui est dite permise ou interdite selon la structure des noyaux en jeu. Les électrons peuvent donc avoir un spectre en énergie continu présentant des formes variées.

Peu de travaux expérimentaux ont été menés sur le sujet depuis les années 1970. Les études se sont concentrées principalement sur les transitions permises et premières interdites non uniques [5]. Mais les utilisateurs sont aussi demandeurs des spectres des transitions interdites uniques et non uniques d'ordres supérieurs.

Un spectre bêta résulte du produit d'une constante de couplage pour l'interaction faible, d'un facteur statistique d'espace des phases, de la fonction de Fermi qui corrige les effets coulombiens dus au noyau, et d'un facteur de forme. Le facteur d'espace des phases reflète simplement la répartition de l'impulsion totale entre l'électron et le neutrino. Le facteur de forme contient toutes les dépendances en énergie restantes, tels les éléments de matrice leptoniques et nucléaires ou la correction des effets atomiques. Un programme de calcul de la forme des spectres bêta pour les transitions permises et interdites uniques a déjà été développé au LNHB et décrit par ailleurs [6].

Les effets atomiques jouent un rôle important dans la détermination de la forme des spectres bêta, surtout à basse énergie. Les électrons atomiques masquent partiellement la charge du noyau vue par l'électron bêta : c'est l'effet bien connu d'écrantage. La variation soudaine de la charge nucléaire lors de la désintégration peut induire des excitations atomiques (shake-up) ou des ionisations internes (shake-off), dont les faibles contributions ne sont pas supérieures à $0,1 \%$ de la probabilité d'émission à une énergie quelconque.

L'effet d'échange est également un effet atomique. Il survient lorsqu'un électron bêta est créé directement dans une orbitale atomique du noyau fils, même si cette orbitale était occupée dans le noyau père. Le principe d'exclusion de Pauli n'est pas violé car les états initial et final ne sont pas orthogonaux, la charge du noyau variant d'une unité au cours de la désintégration. L'électron atomique présent initialement est alors éjecté vers un état du continuum et emporte l'énergie de l'électron bêta moins l'énergie de liaison de l'orbitale. En mesurant uniquement les électrons, ce processus ne peut pas être distingué de la désintégration directe vers un état du continuum.

\subsection{Effet d'échange}

Comme exposé en détail dans [7], l'effet d'échange peut s'exprimer comme un facteur de correction modifiant la probabilité d'émission $P$ à une énergie donnée $E$ de l'électron bêta :

$$
\frac{d P}{d E}=\frac{d P^{\mathrm{NE}}}{d E}\left[1+\eta_{\mathrm{ex}}^{\mathrm{T}}(E)\right]
$$

où l'exposant « $\mathrm{NE}$ » signifie «no exchange », et où $\eta_{\mathrm{ex}}^{\mathrm{T}}$ est le facteur d'échange total. Le facteur d'échange nécessite le calcul des fonctions d'ondes radiales de l'électron dans les états liés et dans les états du continuum, ainsi que le calcul de leur recouvrement.

Dans le cas des transitions permises, seuls les électrons bêta créés dans un état lié « $S$ » de l'atome fils contribuent à $\eta_{\mathrm{ex}}^{\mathrm{T}}$. En effet, une transition bêta est classée selon les propriétés des états nucléaires initiaux et finaux à travers les variations de moment angulaire total $\Delta J$ et de parité $\Delta \pi$. Pour une transition permise, la paire électron antineutrino n'emporte pas de moment angulaire orbital. Donc seules les orbitales $n$ s sont accessibles à l'électron bêta, avec $n$ le nombre quantique principal.

Le facteur d'échange total s'écrit alors comme la somme des contributions de chaque orbitale :

$$
\eta_{\mathrm{ex}}^{\mathrm{T}}(E)=\sum_{n} \eta_{\mathrm{ex}}^{n s}(E)+\sum_{\substack{m, n \\ m \neq n}} \mu_{m} \mu_{n}
$$

et le facteur d'échange de la n-ième orbitale est donné par :

$$
\eta_{\mathrm{ex}}^{n s}(E)=f\left(\mu_{n}^{2}-2 \mu_{n}\right)
$$

Les paramètres $\mu_{n}$ et $f$ s'expriment en fonction des fonctions d'ondes radiales de l'électron liées $\left(f_{n, \mathrm{\kappa}}^{b}, g_{n, \mathrm{\kappa}}^{b}\right)$ et continues $\left(f_{\kappa}^{c}, g_{\mathrm{K}}^{c}\right)$ évaluées au rayon du noyau $R$ selon :

$$
\begin{gathered}
\mu_{n}=\left\langle E s^{\prime} \mid n s\right\rangle \frac{g_{n, \kappa}^{b}(R)}{g_{\kappa}^{c}(R)} \\
f=\frac{g_{\kappa}^{c}(R)^{2}}{g_{\kappa}^{c}(R)^{2}+f_{\kappa}^{c}(R)^{2}}
\end{gathered}
$$


Le nombre quantique $\kappa$ vaut -1 pour les orbitales $s$. $\left\langle E s^{\prime} \mid n s\right\rangle$ symbolise le recouvrement entre l'état lié $|n s\rangle$ et l'état du continuum $\left\langle E s^{\prime}\right|$ sur tout l'espace.

Harston [7] souligne que l'effet d'écrantage doit déformer les fonctions d'ondes et peut donc contribuer au recouvrement de façon significative. Il prend cet effet en compte en utilisant des charges nucléaires effectives lors du calcul des fonctions d'ondes radiales. Cela ne concerne pas les fonctions d'ondes évaluées au rayon du noyau, pour lesquelles la charge nucléaire complète doit être utilisée. Suivant cette méthode, nous avons déterminé les charges nucléaires effectives à partir des rayons moyens des orbitales, pris dans $[8,9]$ dans le cas non relativiste (calculs Hartree-Fock), et dans [10] dans le cas relativiste (calculs Dirac-Fock).

Enfin, les fonctions d'ondes radiales de l'électron analytiques ont été prises dans [11] et s'expriment en termes de fonction hypergéométrique confluente. Une bonne précision sur le calcul du recouvrement nécessite d'évaluer cette fonction spéciale pour des arguments complexes de grands modules. Le formalisme de l'effet d'échange et les calculs résultants ont été détaillés dans [12].

\subsection{Effet d'écrantage}

Lors du calcul d'un spectre bêta, l'effet d'écrantage est généralement corrigé en utilisant un potentiel constant de Thomas-Fermi qui est soustrait de l'énergie totale de la particule [13]. Cette méthode crée une discontinuité non physique à l'énergie minimale définie par ce potentiel [6]. Cette énergie minimale est inférieure ou de l'ordre de $20 \mathrm{keV}$, de telle sorte que la discontinuité n'affecte pas l'utilisation pratique du spectre calculé dans la plupart des cas. Physiquement, l'influence des électrons atomiques est significative lorsque la longueur d'onde de l'électron bêta est comparable à la taille de l'atome, c'està-dire à basse énergie.

Comme cela a été montré dans [12], cette méthode simple, qui permet l'utilisation de fonctions d'ondes analytiques, n'est pas adaptée aux radionucléides de $Z$ élevé et à basse énergie. La méthode usuelle est de corriger le potentiel coulombien du noyau de l'influence des électrons atomiques. De tels potentiels écrantés peuvent être trouvés dans [14]. Cependant, les équations de Dirac auxquelles obéissent les fonctions d'ondes ne peuvent plus être résolues analytiquement. Nous avons donc suivi la méthode numérique décrite en détail dans [15]. Les fonctions d'ondes radiales de l'électron sont développées localement en séries entières, permettant de résoudre les équations de Dirac à l'aide de relations de récurrence. Un traitement spécial est nécessaire pour les solutions relatives au point singulier régulier $r=0$ et au point singulier irrégulier $r=\infty$. Le calcul consiste alors à évaluer les fonctions d'ondes au voisinage de $r=0$, en un point suffisamment éloigné du noyau pour une évaluation correcte de l'expansion asymptotique, et pas à pas entre ces deux points afin de reconnecter chaque solution avec la normalisation et le décalage de phase appropriés.
Pour vérifier nos calculs, nous avons recalculé des tables de paramètres variés, traditionnellement utilisées pour calculer des spectres bêta [16]. Malgré un manque d'informations précises et détaillées sur la manière exacte de calculer ces paramètres, les fonctions de Coulomb non écrantées (Table 2 dans [16]) sont parfaitement reproduites, et les ratios des fonctions de Coulomb écrantées sur non écrantées (Table 3 dans [16]) sont en très bon accord quel que soit $Z$.

Les spectres bêta sont usuellement calculés à partir de la fonction d'onde radiale de l'électron évaluée à la surface du noyau. Pour tenir compte de l'effet d'écrantage, renormaliser les fonctions d'ondes et les évaluer au rayon du noyau est insuffisant car les potentiels écrantés sont très faibles à cette distance, et donc la modification engendrée est complètement négligeable sur l'ensemble du spectre en énergie. En réalité, il est indispensable de tenir compte de l'extension spatiale des fonctions d'ondes. Comme décrit dans [15], cela peut être fait en évaluant la matrice de transition correspondant à la désintégration bêta. Cependant, les calculs deviennent alors très complexes car la fonction d'onde de l'électron est couplée avec les éléments de matrice nucléaires en jeu. Pour contourner cette difficulté, nous pouvons tirer profit du paramètre $f$ défini à l'équation (5) pour l'effet d'échange. En effet, ce paramètre représente la fraction des électrons bêta qui émergent dans un état du continuum $\kappa$ si l'effet d'échange est omis. Plutôt que le carré des fonctions d'ondes évaluées à la surface du noyau, nous avons utilisé la valeur moyenne du carré des fonctions d'ondes radiales sur tout l'espace. C'est-à-dire :

$$
\overline{g_{\kappa}^{2}}=\frac{1}{\Delta R} \int_{\Delta R} g_{\kappa}^{2}(r) d r
$$

où $\Delta R$ est l'intervalle effectif d'intégration. Le facteur corrigeant l'effet d'écrantage est alors :

$$
C_{\mathrm{sc}}=1+\frac{\Delta R_{\mathrm{unsc}}}{\Delta R_{\mathrm{sc}}} \frac{f_{\mathrm{sc}}-f_{\mathrm{unsc}}}{f_{\mathrm{unsc}}}
$$

où l'indice « sc» (respectivement « unsc ») signifie que les fonctions d'ondes écrantées (respectivement non écrantées) ont été utilisées. De cette manière, la correction d'écrantage a une signification physique et est correcte tant que l'on ne traite pas des transitions interdites non uniques. En effet, pour les transitions permises et interdites uniques, les éléments de matrice nucléaires peuvent être factorisés et ne présentent pas de dépendance en énergie de l'électron bêta [6].

\section{Mesure des spectres bêta avec des calorimètres métalliques magnétiques}

Les calorimètres métalliques magnétiques (MMC) sont des détecteurs cryogéniques pouvant être configurés de façon à offrir un rendement de détection très élevé, combiné à une excellente résolution en énergie et un seuil en énergie très bas. 


\subsection{Principe de fonctionnement des MMC}

Comme la plupart des détecteurs cryogéniques [17], les MMC [18] sont des détecteurs thermiques. L'énergie d'une particule incidente est cédée à l'absorbeur du détecteur et entraîne une élévation de température du détecteur. Cette élévation de température est mesurée à l'aide d'un thermomètre en fort contact thermique avec l'absorbeur. Un faible lien thermique entre le détecteur et un bain thermostaté ramène le détecteur à sa température d'équilibre après chaque interaction d'une particule. Le signal d'un calorimètre magnétique est donc une impulsion de température pour chaque particule détectée, contrairement aux détecteurs fonctionnant selon un mode bolométrique, qui mesurent une puissance incidente et ne peuvent pas mesurer des particules individuelles.

L'élévation de température $\Delta T$ du détecteur est proportionnelle à l'énergie $E$ cédée par la particule et inversement proportionnelle à la capacité thermique $C \mathrm{du}$ détecteur (absorbeur + thermomètre) $: \Delta T=E / C$. Elle devient mesurable à très basse température $(<100 \mathrm{mK})$, puisque les capacités thermiques de la plupart des matériaux ainsi que les bruits thermiques diminuent avec la température.

Les absorbeurs des calorimètres magnétiques sont généralement métalliques, le plus souvent en or. Ce métal offre un pouvoir d'arrêt élevé. Dans le contexte de la spectrométrie bêta, où les sources sont déposées sur le matériel de l'absorbeur (partie 3.2.), la bonne résistance chimique de l'or est un avantage supplémentaire.

À très basse température, la contribution principale à la capacité thermique d'un métal est celle des électrons de conduction. L'énergie d'une particule incidente est transférée à l'ensemble des électrons de conduction en une durée de l'ordre de la microseconde. Cette durée est principalement déterminée par la diffusivité thermique et les dimensions de l'absorbeur. Les thermomètres des calorimètres magnétiques sont réalisés dans un alliage paramagnétique d'or contenant une faible concentration (de l'ordre de un pour mille) d'erbium (Au:Er). La présence d'un champ magnétique (quelques milliteslas) induit une aimantation du thermomètre due à l'orientation des spins des ions d'erbium $\mathrm{Er}^{3+}$. Cette aimantation varie fortement avec la température. L'élévation de température du détecteur se traduit donc par une variation de l'aimantation du thermomètre, qui peut être lue à l'aide d'un SQUID (Superconducting Quantum Interference Device) [19], un magnétomètre très sensible.

Le temps de transfert de chaleur entre l'absorbeur et le thermomètre dépend de la nature du contact entre ces deux éléments. Si le contact est métallique, l'équilibre thermique entre l'absorbeur et le thermomètre s'établit dans un temps de l'ordre de $1 \mu$ s. L'équilibre thermique entre les électrons de conduction et les spins d'erbium dans le thermomètre est très rapide, inférieur à $1 \mu \mathrm{s}$. Le temps de montée intrinsèque des impulsions des calorimètres magnétiques est donc habituellement de quelques microsecondes.

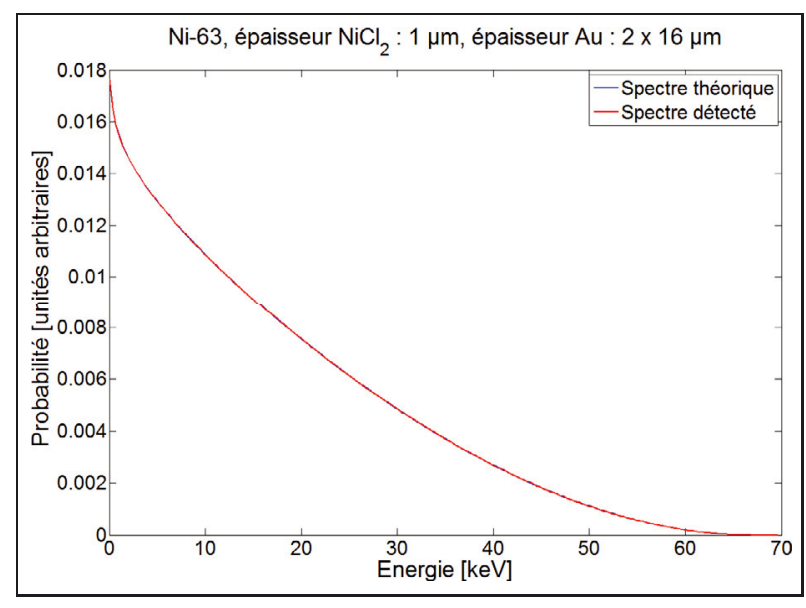

Fig. 1. - Simulation de Monte-Carlo du spectre bêta du ${ }^{63} \mathrm{Ni}$ détecté par un calorimètre métallique magnétique comportant un absorbeur en or (épaisseur $(2 \times 16) \mu \mathrm{m})$ qui enferme la source de ${ }^{63} \mathrm{Ni}$.

Le temps de retour à la température d'équilibre, ou temps de décroissance des impulsions, est donné par $\tau=C / G$, où $G$ est la conductivité thermique du lien entre le détecteur et le bain thermostaté. La constante de temps et le taux de comptage peuvent donc être ajustés par le lien thermique. Dans la plupart des cas, un temps de décroissance de l'ordre de la milliseconde est souhaitable. Des temps de décroissance trop courts conduisent à une dégradation de la résolution en énergie du détecteur; des temps de décroissance plus longs réduisent le taux de comptage maximal.

Les meilleures résolutions en énergie et les plus faibles seuils de détection en énergie sont atteints pour une température du détecteur située entre $10 \mathrm{mK}$ et $30 \mathrm{mK}$.

\subsection{MMC pour la spectrométrie bêta}

Pour une mesure précise de la forme des spectres bêta, le détecteur devrait idéalement offrir un angle solide de $4 \pi$ sr et un rendement de détection intrinsèque de $100 \%$. Le MMC avec l'émetteur bêta enfermé dans l'absorbeur répond très bien à ces exigences. L'angle solide de $4 \pi$ sr résulte du simple fait d'enfermer la source dans l'absorbeur, et celui-ci peut être dimensionné, à l'aide d'une méthode de Monte-Carlo, de telle sorte que tous les électrons bêta soient complètement arrêtés. Ces simulations permettent également de vérifier que le spectre en énergie n'est pas déformé par l'éventuel échappement de photons de Bremsstrahlung. La figure 1 montre le résultat d'une simulation effectuée avec le code PENELOPE [20], pour un MMC développé pour la mesure du spectre du ${ }^{63} \mathrm{Ni}$. Le spectre théorique, calculé en tenant compte des effets d'échange et d'écrantage (cf. partie 2), a été utilisé comme donnée d'entrée de la simulation. Le spectre simulé correspond à l'énergie déposée suite à chaque désintégration dans l'ensemble de l'absorbeur incluant le sel $\left(\mathrm{NiCl}_{2}\right)$ formant la source. La superposition des deux spectres est parfaite : le spectre théorique 


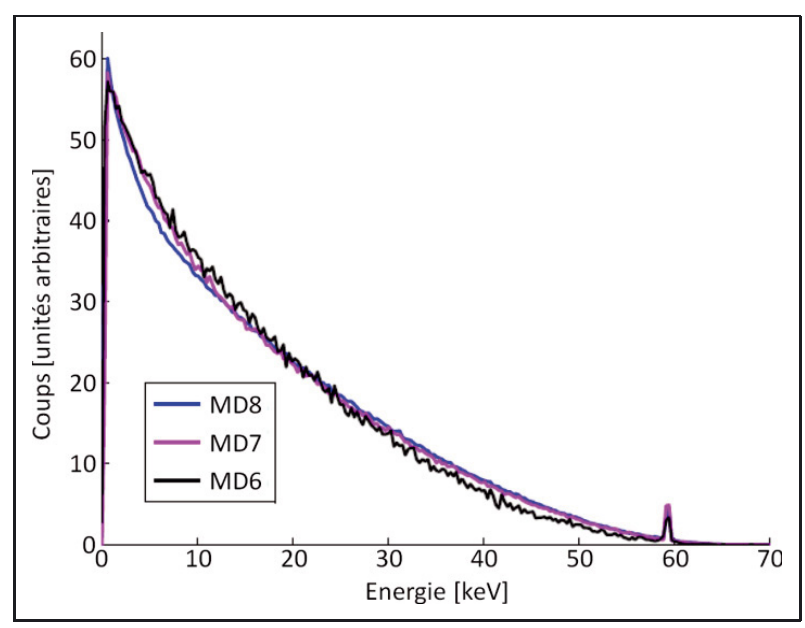

Fig. 2. - Spectre bêta du ${ }^{63} \mathrm{Ni}$ mesuré avec des sources déposées à partir d'une solution de $\mathrm{NiCl}_{2}$ et enfermées dans les absorbeurs de calorimètres métalliques magnétiques (appelés MD6, MD7 et MD8 respectivement). Le pic à 59,5 keV provient des photons gamma d'une source de ${ }^{241} \mathrm{Am}$ utilisée pour l'étalonnage en énergie. La linéarité des détecteurs a été vérifiée dans des mesures dédiées, utilisant des sources de photons entre $5,9 \mathrm{keV}$ et $88 \mathrm{keV}$.

est quasi invisible. Si le taux de comptage est assez faible compte tenu de la constante de temps thermique du détecteur, le rendement de détection est de $100 \%$ entre le seuil de détection en énergie et l'énergie maximale du spectre. Les absorbeurs des MMC développés pour la mesure des spectres $\mathrm{du}{ }^{63} \mathrm{Ni}$ ainsi que $\mathrm{du}{ }^{241} \mathrm{Pu}$ ont des dimensions d'environ $0,7 \mathrm{~mm} \times 0,7 \mathrm{~mm} \times(2 \times 16) \mu \mathrm{m}$ et une capacité thermique de l'ordre de $10 \mathrm{pJ} \cdot \mathrm{K}^{-1}$ à $10 \mathrm{mK}$. Les résolutions en énergie varient entre $30 \mathrm{eV}$ et $50 \mathrm{eV}$ (FWHM) pour des énergies jusqu'à $60 \mathrm{keV}$, les seuils de détection en énergie entre $200 \mathrm{eV}$ et $300 \mathrm{eV}$.

\subsection{Influence de la qualité de la source bêta sur la forme du spectre mesuré}

Un avantage apparent des MMC enfermant la source bêta est que l'énergie déposée par les électrons bêta dans la source («auto-absorption ») reste confinée dans le détecteur et devrait être détectée. Or, la forme mesurée du spectre du ${ }^{63} \mathrm{Ni}$ en utilisant différentes sources déposées à partir d'une solution de $\mathrm{NiCl}_{2}$ [21] n'est pas reproductible, comme l'illustre la figure 2.

À part le dépôt de $\mathrm{NiCl}_{2}$ des sources, dont la cristallisation varie d'un échantillon à l'autre, les MMC utilisés dans ces mesures sont quasi identiques. Ce fait et la vérification de la linéarité des MMC nous ont permis de conclure que les écarts entre les spectres doivent être attribués aux sources. L'explication la plus plausible est qu'une partie de l'énergie déposée dans le sel formant la source est transformée en états métastables (e.g. paires électron-trou) et n'est pas thermalisée [22], du moins sur une échelle de temps comparable à la durée des impulsions. Cette partie de l'énergie ne serait donc pas mesurée sous forme de chaleur par le thermomètre.

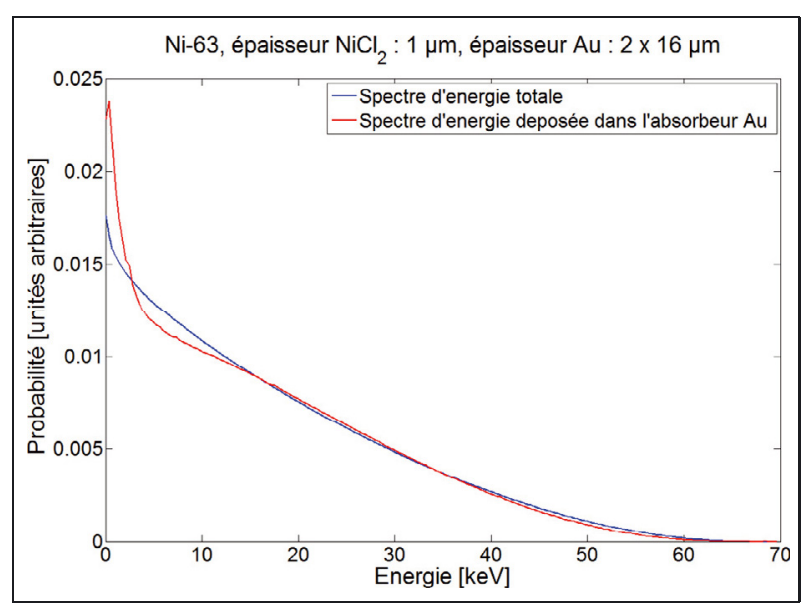

Fig. 3. - Simulation de Monte-Carlo du spectre bêta du ${ }^{63} \mathrm{Ni}$ détecté par un calorimètre métallique magnétique comportant un absorbeur en or (épaisseur $(2 \times 16) \mu \mathrm{m})$ qui enferme une source de ${ }^{63} \mathrm{Ni}\left(\mathrm{NiCl}_{2}\right)$ d'épaisseur $1 \mu \mathrm{m}$. Le spectre bleu représente l'énergie déposée dans l'ensemble source-absorbeur et est identique au spectre théorique. Le spectre rouge correspond à l'énergie des électrons sortant de la source et déposée dans l'absorbeur en or.

La figure 3 montre le résultat d'une simulation de Monte-Carlo de l'énergie déposée dans l'absorbeur en or, le reste de l'énergie étant cédé à la source. La simulation considère une épaisseur supposée de la source $\left(\mathrm{NiCl}_{2}\right)$ de $1 \mu \mathrm{m}$, ce qui correspondrait au séchage sur une surface d'un diamètre de quelques dizaines de micromètres. La vraie épaisseur des sources est difficile à estimer car le séchage se fait de façon non uniforme, mais nous avons observé des dépôts de cet ordre de grandeur. Des essais de détermination quantitative de l'épaisseur du dépôt n'ont pas abouti, ni le profilage par un microscope interférométrique de la surface de la feuille d'or avec le dépôt de $\mathrm{NiCl}_{2}$, ni la diffractométrie de rayons $\mathrm{X}$, en raison d'une trop grande rugosité de la surface. La simulation peut donc seulement être considérée qualitativement. Elle représente par ailleurs la situation extrême dans laquelle toute l'énergie déposée dans la source serait perdue pour la détection. La réalité se trouve entre le spectre déposé dans l'or, en rouge, et le spectre déposé dans l'ensemble source-absorbeur, en bleu. Mais cette simulation permet de confirmer que l'explication d'une déformation des spectres telle qu'observée en figure 2 par la non-transformation d'une partie de l'énergie en chaleur est plausible.

Suite aux difficultés rencontrées avec les sources réalisées par séchage d'une solution de $\mathrm{NiCl}_{2}$, une source a été fabriquée par électrodéposition sur une feuille d'or, donnant un dépôt métallique de Ni [3]. Cette feuille a été intercalée entre deux feuilles d'or (épaisseur de $16 \mu \mathrm{m}$ ) formant l'absorbeur. L'électrodéposition a été choisie parce que des états métastables sous forme de paires électron-trou n'existent pas dans un métal. Les résultats présentés en partie 4.1 ont été obtenus avec cette source électrodéposée et confirment que ce type de source est adapté à la mesure de l'énergie des électrons bêta sous forme de chaleur. 


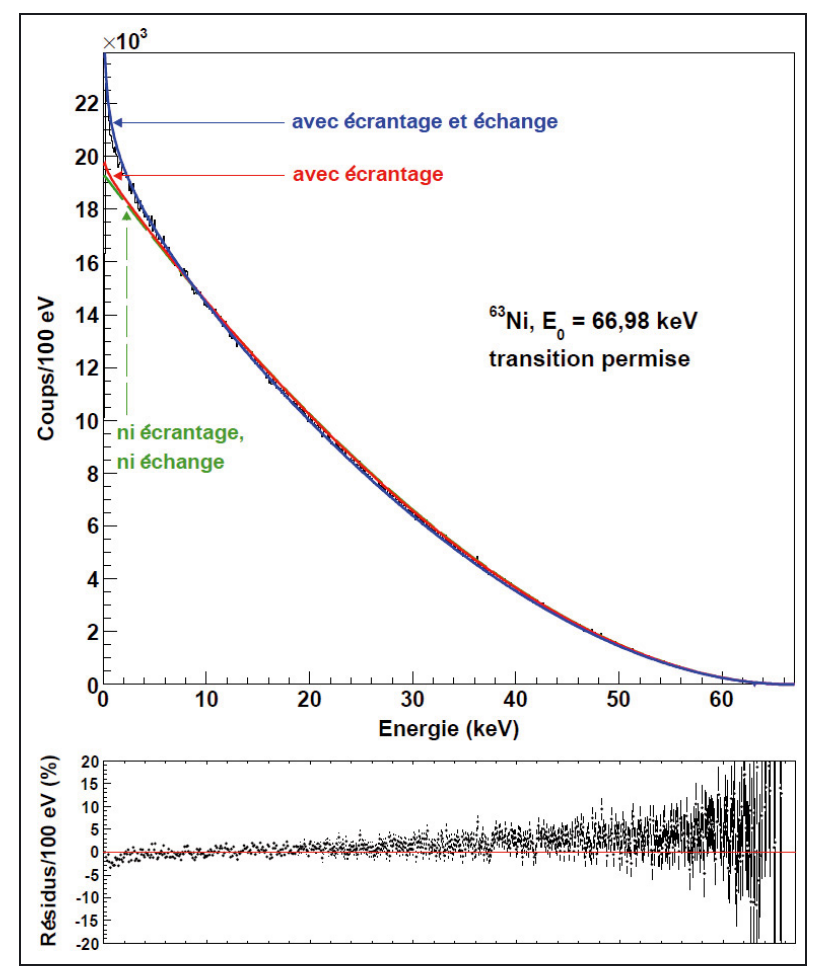

Fig. 4. - Comparaison entre le spectre bêta du ${ }^{63} \mathrm{Ni}$ mesuré [3] et le spectre théorique calculé de trois manières : le calcul classique pour une transition permise sans écrantage ni effet d'échange, le même calcul en corrigeant de l'écrantage, et enfin le calcul complet tenant compte de l'écrantage et de l'effet d'échange. Les résidus correspondant au spectre théorique complet par rapport au spectre mesuré sont présentés dans la partie basse de la figure.

\section{Résultats}

Les spectres bêta mesurés pour le ${ }^{63} \mathrm{Ni}$ [3] et le ${ }^{241} \mathrm{Pu}$ [4] ont été comparés dans un premier temps à des calculs classiques. Une déviation systématique du spectre expérimental à basse énergie (en dessous de $8 \mathrm{keV}$ pour le ${ }^{63} \mathrm{Ni}$, et en dessous de $4 \mathrm{keV}$ pour le ${ }^{241} \mathrm{Pu}$ ) par rapport à ces calculs n'a pas pu être expliquée.

\section{1. $\quad{ }^{63} \mathrm{Ni}$}

Le radionucléide ${ }^{63} \mathrm{Ni}$ décroît entièrement par émission $\beta^{-}$vers l'état fondamental du ${ }^{63} \mathrm{Cu}$. La nature de la transition est permise, avec une énergie maximale de $66,980(15) \mathrm{keV}$ et une période de 98,7(24) ans [23].

Les calculs des facteurs d'échange partiels et total ont été effectués avec des charges nucléaires effectives non relativistes, mais les résultats sont identiques avec des charges effectives relativistes. En cohérence avec la composition chimique de la source, nous avons considéré un atome neutre de nickel ayant ses orbitales 1 s à 4 s complètement remplies. Le facteur d'échange total est en très bon accord avec celui donné de $5 \mathrm{keV}$ à $67 \mathrm{keV}$ dans [24], où malheureusement aucun détail sur les calculs n'est fourni. Le calcul de la correction d'écrantage a été effectué avec le potentiel d'écrantage donné dans [14]. Les spectres résultants sont comparés avec le spectre mesuré de [3] en figure 4.
Comme attendu, l'influence de l'effet d'écrantage est faible pour ce radionucléide de faible $Z$, mais l'influence de l'effet d'échange induit une énergie moyenne du spectre bêta inférieure de 2,6\% à celle déterminée sans tenir compte de ces phénomènes.

\section{2. $\quad{ }^{241} \mathrm{Pu}$}

Le radionucléide ${ }^{241} \mathrm{Pu}$ décroît essentiellement par désintégration $\beta^{-}$vers l'état fondamental de ${ }^{241} \mathrm{Am}$ avec une probabilité de transition évaluée à 99,997 56(2)\%, le reste étant de la désintégration alpha [25]. La nature de la transition est première interdite non unique, avec une énergie maximale de $20,8(2) \mathrm{keV}$ et une période de $14,33(4)$ ans.

Dans le cas des transitions interdites non uniques, la dépendance en énergie des éléments de matrice nucléaires ne peut pas être factorisée, ce qui complique grandement les calculs de la forme de ces spectres d'énergie bêta. Cependant, les transitions premières interdites non uniques qui satisfont à l'approximation $2 \xi=$ $\alpha Z / R \gg E_{0}$ peuvent être traitées comme des permises [26], avec $\alpha$ la constante de structure fine, $R$ le rayon du noyau, $Z$ sa charge, et $E_{0}$ l'énergie maximale de la transition. Cette approximation, dite approximation $\xi$, signifie simplement que l'énergie coulombienne de l'électron bêta à la surface du noyau doit être très grande devant l'énergie totale disponible dans la transition. La décroissance du ${ }^{241} \mathrm{Pu}$ est un cas d'école pour l'approximation $\xi$ à cause d'un $E_{0}$ faible comparé à $2 \xi \approx$ 19,8 MeV. La précision de l'approximation $\xi$ est d'environ $E_{0} / \xi$, de l'ordre de $0,1 \%$ ici. De plus, le facteur de forme de cette transition a déjà été étudié par un calcul complet des éléments de matrice nucléaires dans le cadre du modèle de Nilsson [27]. Son influence croît linéairement avec l'énergie, mais ne dépasse pas $0,3 \%$ à l'énergie maximale et $0,1 \%$ en dessous de $8 \mathrm{keV}$. Par conséquent, cette transition peut être calculée comme une transition permise.

La correction d'effet d'échange a été calculée initialement dans les mêmes conditions que celles données dans [7], soit avec les mêmes charges nucléaires effectives et en considérant un ion $\mathrm{Pu}^{3+}$ dont les orbitales atomiques $1 \mathrm{~s}$ à $6 \mathrm{~s}$ sont complètement remplies. Nos calculs sont en très bon accord avec les résultats présentés dans [7]. En cohérence avec la composition chimique de la source utilisée dans [4], nous avons considéré dans un deuxième temps un atome neutre de plutonium, dont l'orbitale $7 \mathrm{~s}$ est elle aussi remplie et qui a une contribution significative en dessous de $1 \mathrm{keV}$. Bien évidemment, il est nécessaire d'utiliser des charges nucléaires relativistes pour ce noyau de grand $Z$.

Le calcul de la correction d'écrantage a été effectué avec le potentiel d'écrantage donné dans [14]. Les spectres résultants sont comparés avec le spectre mesuré de [4] en figure 5.

Pour ce radionucléide de Z élevé, l'effet d'écran a une contribution significative, équivalente à celle de l'effet 


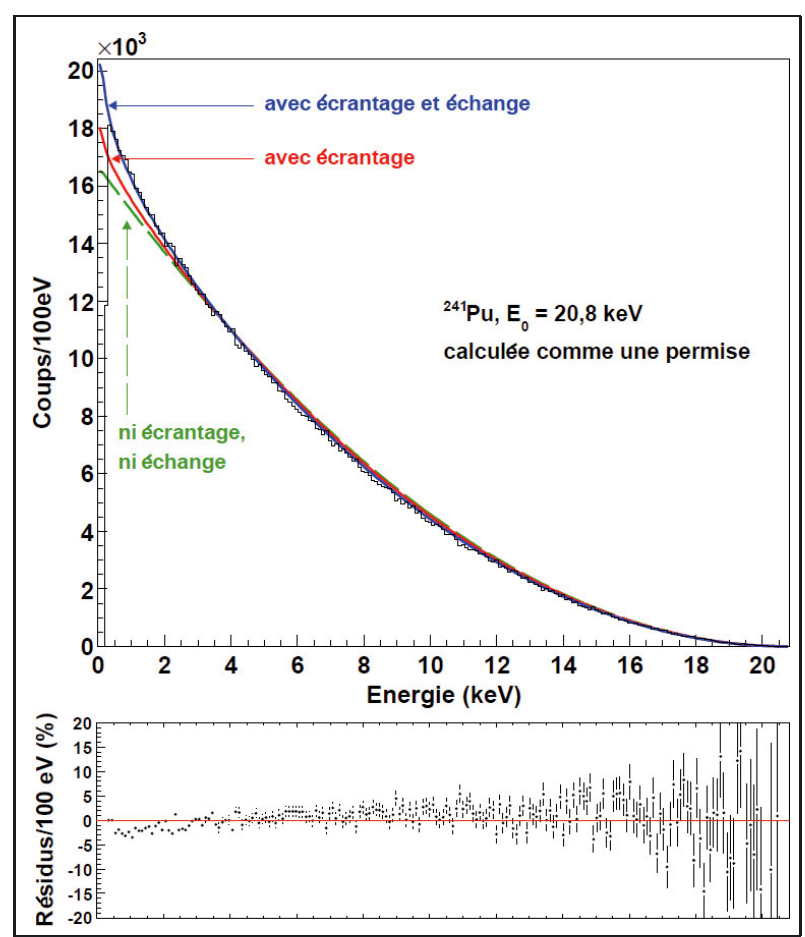

Fig. 5. - Comparaison entre le spectre bêta du ${ }^{241} \mathrm{Pu}$ mesuré [4] et le spectre théorique calculé de trois manières : le calcul classique pour une transition permise sans écrantage ni effet d'échange, le même calcul en corrigeant de l'écrantage, et enfin le calcul complet tenant compte de l'écrantage et de l'effet d'échange. Les résidus correspondant au spectre théorique complet par rapport au spectre mesuré sont présentés dans la partie basse de la figure.

d'échange. Lorsque ces deux effets sont pris en compte l'énergie moyenne résultante est de 3,5\% inférieure à celle calculée sans ces corrections.

\section{Conclusion}

Les spectres en énergie de l'émission bêta du ${ }^{63} \mathrm{Ni}$ et $\mathrm{du}{ }^{241} \mathrm{Pu}$, qui ont récemment été mesurés avec des calorimètres métalliques magnétique, sont bien reproduits par nos calculs jusqu'aux très basses énergies. Il est démontré que l'effet d'échange a une influence majeure sur la forme du spectre dans cette gamme d'énergie.

L'influence des effets d'écrantage et d'échange sur la forme du spectre et sur l'énergie moyenne a un impact direct sur la mesure d'activité de ces nucléides. Le spectre expérimental du ${ }^{241} \mathrm{Pu}$ de [4] a été utilisé par plusieurs laboratoires nationaux de métrologie, qui ont pu améliorer les incertitudes de mesure d'activité de ${ }^{241} \mathrm{Pu}$ et/ou résoudre des écarts entre différentes méthodes de mesure par scintillation liquide [28-31]. Pour la mesure d'activité du ${ }^{63} \mathrm{Ni}$ par la méthode RCTD (Rapport des Coïncidences Triples à Doubles), qui est la méthode de référence pour ce nucléide, un biais relatif entre $0,5 \%$ et $1 \%$ par l'omission de l'effet d'échange dans le calcul du spectre a été estimé, en considérant différentes conditions expérimentales. Ce biais est important compte tenu de l'incertitude type relative d'environ $0,5 \%$ pour les mêmes conditions de mesure.
On peut observer, dans les résidus, une faible déviation systématique pour ces deux transitions. Elle est probablement due au fait que les fonctions d'ondes des états liés et des états du continuum impliquées dans l'effet d'échange sont évaluées par des expressions analytiques à l'aide de charges nucléaires effectives. De plus, ces charges effectives ne sont pas déterminées, à ce stade, avec les mêmes potentiels que pour l'effet d'écrantage. Nous élaborons actuellement un nouveau programme pour calculer l'effet d'échange avec des fonctions d'ondes identiques à celles utilisées pour l'écrantage.

Nous avons observé que la justesse et la reproductibilité des spectres mesurés dépendent fortement de la qualité des sources bêta, du moins pour les émetteurs bêta de basse énergie. Les sources électrodéposées se sont avérées bien adaptées à la mesure de l'énergie des particules bêta sous forme de chaleur. Pour des nucléides ne pouvant pas être électrodéposés, des études de la meilleure méthode de fabrication de sources doivent être menées. Des sources implantées dans l'absorbeur du détecteur seraient idéales, mais très peu d'installations capables d'implanter des radionucléides existent.

\section{Références}

[1] International Atomic Energy Agency (IAEA), "Final report: Focused workshop on antineutrino detection for safeguards applications", IAEA Headquarters, Vienna, 2008.

[2] KASsis A.I., "The amazing world of Auger electrons", Int. J. Rad. Biol., 80, 2004, 789-803.

[3] LE-BRET C., « Développement de calorimètres métalliques magnétiques pour la spectrométrie bêta », Thèse de doctorat, Université Paris Sud (Paris 11), 7 septembre 2012.

[4] Loidl M., Rodrigues M., Censier B., Kowalski S., Mougeot X., CAssette P., Branger T. et Lacour D., "First measurement of the beta spectrum of ${ }^{241} \mathrm{Pu}$ with a cryogenic detector", Appl. Rad. Isotopes, 68, 2010, 14541458.

[5] Gorozhankin V., BÉ M.-M., Mougeot X., PerevoshchiKov L. et BRIANÇON Ch., "Construction of beta spectrum on the basis of experimental nuclear decay data", Proceedings of the LSC2010 International Conference, édité par P. Cassette, University of Arizona, septembre 2010, 259-266.

[6] Mougeot X., BÉ M.-M., Chisté V., Dulieu C., GOROZHANKIN V. et LOIDL M., "Calculation of beta spectra for allowed and unique forbidden transitions", Proceedings of the 2010 International Conference on Advances in Liquid Scintillation Spectrometry (LSC 2010), 6-10 sept. 2010, Paris, France, Ed. P. Cassette, Radiocarbon, 2011, 249-257.

[7] HaRston M.R. et PyPER N.C., "Exchange effects in beta decays of many-electron atoms", Phys. Rev. A, 45, 1992, 6282-6295. 
[8] FISCHER C.F., "The Hartree-Fock method for atoms", Wiley and Sons, 1977.

[9] MANN J.B., "SCF Hartree-Fock results for elements with two open shells and for the elements Francium to Nobelium", At. Data Nucl. Data Tables, 12, 1973, 1-86.

[10] DesclauX J.P., "Relativistic Dirac-Fock expectation values for atoms with $Z=1$ to $Z=120$ ", At. Data Nucl. Data Tables, 12, 1973, 311-406.

[11] Rose M.E., "Relativistic electron theory", Wiley and Sons, 1961.

[12] Mougeot X., BÉ M.-M., BISch C. et LoIDL M., "Evidence for the exchange effect in the beta decay of ${ }^{241} \mathrm{Pu}$ ", Phys. Rev. A, 86, 2012, 042506 1-7.

[13] Good Jr. R.H., "Effect of atomic electron screening on the shape of forbidden beta spectra", Phys. Rev., 94, 1954 931-933.

[14] Salvat F., Martílnez J., Mayol R. et Parellada J., "Analytical Dirac-Hartree-Fock-Slater screening function for atoms $(Z=1-92)$ ", Phys. Rev. A, 36, 1987, 467-474.

[15] BEHRENS H. et BÜHRING W., "Electron radial wave functions and nuclear beta decay", Oxford Science Publications, 1982

[16] BEHRENS H. et JÄNECKE J., "Numerical tables for betadecay and electron capture", Landolt-Börnstein, New Series, Group I, Vol. 4, Springer Verlag, Berlin, 1969.

[17] Proceedings of the $15^{\text {th }}$ International Workshop on Low Temperature Detectors (LTD-15), Pasadena, États-Unis, 24-28 juin 2013, J. Low Temp. Phys., 176, 2014.

[18] Fleischmann A., Enss C. et Seidel G., « Metallic Magnetic Calorimeters », dans « Cryogenic Particle Detectors », Enss, C. (Ed.), Topics in Applied Physics, 99, Springer Berlin/Heidelberg, 2005, 151-216.

[19] Clarke J. et BRAginsKi A.I., "The SQUID Handbook", Wiley and Sons, 2004.

[20] Salvat F. et al., "PENELOPE - A code system for Monte Carlo simulation of Electron and Photon transport”, Rapport NEA/NSC/DOC, 2001, 19.

Article reçu le 29 novembre 2013, version révisée reçue le 7 novembre 2014.
[21] Le-Bret C., Loidl M., Rodrigues M., Mougeot X et BOUCHARD J., "Study of the influence of the source quality on the determination of the shape factor of beta spectra", J. Low Temp. Phys., 167, 2012, 985-990.

[22] McCammon D., Juda M., Zhang J., Kelley R.L., Moseley S.H. et SZYMKowiaK A.E., "Thermal detectors for high resolution spectroscopy", IEEE Trans. Nucl. Sci., 33, 1985, 236-239.

[23] Bé M.-M., Chisté V., Dulieu C., Browne E., Baglin C., Chechev V., Kuzmenco N., Helmer R., KondeV F., MacMahon D. et LEE K.B., "Table of Radionuclides", Monographie BIPM-5, Vol. 3, éditée par CEA/LIST-LNHB, BIPM, 2006, 29.

[24] AngRaVE L., "The physics of non-equilibrium phonons and non-equilibrium superconductivity applied to a precision measurement of the beta spectrum of ${ }^{63} \mathrm{Ni}$ ", Thèse de doctorat, Magdalen College, University of Oxford, 2000.

[25] BÉ M.-M., Chisté V., Dulieu C., Browne E., CheCheV V., Kuzmenco N., Kondev F., Luca A., Galán M., PEARCE A et HuANG X, "Table of Radionuclides", Monographie BIPM-5, Vol. 4, éditée par CEA/LISTLNHB, BIPM, 2008, 259.

[26] SCHOPPER H.F., "Weak interactions and nuclear beta decay", North-Holland Publishing Company, 1966.

[27] RizeK J., RYSAVy M. et BRABEC V., "Effect of nuclear structure on the single particle beta minus transitions in deformed nuclei", Czechoslovak J. Phys., 45, 1995, 477-489.

[28] BERGERON D.E. et ZIMMERMAN B.E., "TDCR measurements on ${ }^{241} \mathrm{Pu}$ at NIST", Proceedings of the 2010 International Conference on Advances in Liquid Scintillation Spectrometry (LSC 2010), 6-10 sept. 2010, Paris, France. Ed. P. Cassette, Radiocarbon, 2011, 171-179.

[29] Kossert K., NÄHLE O.J. et Grau Carles A., "Beta shape-factor function and activity determination of ${ }^{241} \mathrm{Pu}$ ", Appl. Rad. Isotopes, 69, 2011, 1246-1250.

[30] VAN WyngaARdT W.M., Simpson B.R.S., VAN STADEN M.J. et LUBBE J., "Absolute standardization of ${ }^{241} \mathrm{Pu}$ by the TDCR technique and effect of the beta spectral shape", Appl. Rad. Isotopes, 70, 2012, 2188-2194.

[31] MichotTe C. et Johansson L., "CCRI(II) activity comparison of ${ }^{241} \mathrm{Pu}$ CCRI(II)-K2.Pu-241", Metrologia 49, 2012, Tech. Suppl., 06012. 